\title{
Long-Lasting Adaptations of the NR2B-Containing NMDA Receptors in the Dorsomedial Striatum Play a Crucial Role in Alcohol Consumption and Relapse
}

\author{
Jun Wang, ${ }^{1}$ Maria Fe Lanfranco, ${ }^{1}$ Stuart L. Gibb, ${ }^{1}$ Quinn V. Yowell, ${ }^{1}$ Sebastien Carnicella, ${ }^{1,2 *}$ and Dorit Ron ${ }^{1,2 *}$ \\ ${ }^{1}$ Ernest Gallo Research Center and ${ }^{2}$ Department of Neurology, University of California, San Francisco, Emeryville, California 94608
}

\begin{abstract}
A growing number of studies suggest that the development of compulsive drug seeking and taking depends on dorsostriatal mechanisms. We previously observed that ex vivo acute exposure of the dorsal striatum to, and withdrawal from, alcohol induces long-term facilitation (LTF) of the activity of NR2B-containing NMDA receptors (NR2B-NMDARs) in a mechanism that requires the Src family protein tyrosine kinase (PTK), Fyn (Wang et al., 2007). In the present study, we first compared alcohol's actions in rat dorsomedial (DMS) and the dorsolateral (DLS) subregions of the striatum, which differ in their anatomical connectivity and function. We found that alcoholmediated induction of LTF of NR2B-NMDAR activity is centered in the DMS. Next, we tested whether in vivo exposure of rats to alcohol leads to long-term adaptations of the NMDAR system in the DMS. We observed that repeated daily administration of alcohol results in a long-lasting increase in the activity of the NR2B-NMDARs in the DMS. The same procedure leads to a prolonged activation of Fyn, increased NR2B phosphorylation, and membrane localization of the subunit. Importantly, similar electrophysiological and biochemical modifications were observed in the DMS of rats that consumed large quantities of alcohol. Finally, we show that inhibition of NR2BNMDARs or Src family PTKs in the DMS, but not in the DLS, significantly decreases operant self-administration of alcohol and reduces alcohol-priming-induced reinstatement of alcohol seeking. Our results suggest that the upregulation of NR2B-NMDAR activity within the DMS by alcohol contributes to the maladaptive synaptic changes that lead to excessive alcohol intake and relapse.
\end{abstract}

\section{Introduction}

Increasing evidence in recent years suggests that the dorsal striatum plays an important role in the development of compulsive drug seeking and taking (Gerdeman et al., 2003; Everitt and Robbins, 2005; Hyman et al., 2006). The dorsal striatum can be divided into two parts: the DMS and the DLS, which differ in connectivity, receptor distribution, synaptic plasticity, and behavioral functions (Joel and Weiner, 2000; Gerdeman et al., 2003; Voorn et al., 2004; Yin and Knowlton, 2006; Belin et al., 2009). In rodents, the DMS receives inputs primarily from several cortical areas, the medial part of the substantia nigra pars compacta $(\mathrm{SNc})$, the ventral tegmental area, and the basolateral amygdala, whereas the DLS receives inputs from the sensorimotor cortex and the lateral part of the SNc (McGeorge and Faull, 1989; Joel and Weiner, 2000; Reep et al., 2003; Voorn et al., 2004). Moreover, long-term potentiation (LTP) is preferentially expressed in the DMS as opposed to the DLS, in which long-term depression (LTD) is more commonly found (Partridge et al., 2000). Inter-

\footnotetext{
Received May 3, 2010; revised June 16, 2010; accepted June 16, 2010.

This research was supported by the National Institute on Alcohol Abuse and Alcoholism (R01AA/MH13438) (D.R.) and by funds provided by the State of California for medical research on alcohol and substance abuse through the University of California, San Francisco (D.R.). We thank Dr. Greg Hjelmstad for helpful discussions.

*S.C. and D.R. contributed equally to this study.

Correspondence should be addressed to Dr. Dorit Ron, The Gallo Research Center, 5858 Horton Street, Suite 200, Emeryville, CA 94608. E-mail: dron@gallo.ucsf.edu.

S. Carnicella's present address: Grenoble Institute of Neuroscience, INSERM U836, F-38042 Grenoble, France. DOI:10.1523/JNEUROSCI.2268-10.2010

Copyright $(\odot 2010$ the authors $\quad 0270-6474 / 10 / 3010187-12 \$ 15.00 / 0$
}

estingly, greater levels of CB1 cannabinoid receptors, which have been shown to promote LTD (Gerdeman et al., 2002), are found in the DLS than in the DMS (Hohmann and Herkenham, 2000).

In addition, rodent and human data suggest that the DMS is important for the formation and processing of action-outcome (A-O) associations that mediate goal-directed behaviors, whereas the DLS has been implicated in the development of habit, stimulus-response, and learning (O'Doherty et al., 2004; Tricomi et al., 2004; Yin and Knowlton, 2006; Balleine et al., 2007; Tanaka et al., 2008; Balleine et al., 2009; Tricomi et al., 2009). Interestingly, exposure to amphetamine can enhance both goal-directed and habit formation behaviors in rats (Nelson and Killcross, 2006; Nordquist et al., 2007). Furthermore, operant response under a procedure that favors the development of habit formation becomes more rapidly habitual in rats trained to orally selfadminister cocaine or alcohol (ethanol) than with a non-drug reinforcer such as sucrose (Dickinson et al., 2002; Miles et al., 2003). Together, these studies suggest that the DMS and DLS may be involved in different aspects that lead to the development of addiction.

Previously, we found that acute ex vivo exposure of striatal slices to, and withdrawal from, ethanol results in LTF of NR2BNMDAR activity in the dorsal but not the ventral striatum (Wang et al., 2007). Here, we set out to test whether ethanol acts preferentially within a specific subregion of the dorsal striatum to alter the activity of the NMDARs, and determine whether ethanolmediated LTF of NR2B-NMDAR plays a role in mechanisms underlying increased motivation to consume ethanol. 


\section{Materials and Methods}

\section{Reagents}

The monoclonal anti-NR1 and anti-NR2B antibodies, and polyclonal anti[pY1472]NR2B antibodies were obtained from Cell Signaling Technology. The polyclonal anti-NR2A, anti-Fyn, anti-NR2B, anti-Actin, anti-GAPDH, and the secondary antibodies were purchased from Santa Cruz Biotechnologies. The polyclonal anti-[pY418/420]Src/Fyn (for measuring active Fyn) and polyclonal anti-[pY529/531]Src/Fyn (for measuring inactive Fyn) antibodies, NuPAGE Bis-Tris gradient gels, and protein $\mathrm{G}$ agarose were obtained from Invitrogen. Bicinchoninic acid (BCA) protein assay kit was obtained from Pierce. Nitrocellulose membrane was purchased from Millipore. 4-Amino-5-(4-chlorophenyl)-7-(t-butyl)pyrazolo[3,4-D]pyrimidine (PP2) was purchased from Calbiochem. 2,3-Dioxo-6-nitro-1,2,3,4-tetrahydrobenzo [f]quinoxaline-7-sulfonamide disodium salt (NBQX), $(\alpha R, \beta S)-\alpha-(4-$ hydroxyphenyl)- $\beta$-methyl-4-(phenylmethyl)-1-piperidinepropanol hydrochloride (Ro 25-6981), and ifenprodil were obtained from Tocris Bioscience. Sodium orthovanadate, picrotoxin, and phosphatase inhibitor cocktails 1 and 2 were obtained from Sigma. Complete Mini, EDTA-free protease inhibitor cocktail was purchased from Roche. Enhanced Chemiluminescence (ECL) Plus was purchased from GE Healthcare.

\section{Animals}

Male Sprague Dawley rats (3-4 weeks old) and male Long-Evans rats $(300 \mathrm{~g})$ were obtained from Harlan. Animals were housed under a light: dark cycle of $12 \mathrm{~h}$, with lights on at 7:00 A.M. and food and water available ad libitum. All animal procedures in this report were approved by the Gallo Center Institutional Animal Care and Use Committee and were conducted in agreement with the Guide for the Care and Use of Laboratory Animals, National Research Council, 1996.

\section{In vivo ethanol administration}

Sprague Dawley rats were injected intraperitoneally with ethanol $(20 \% \mathrm{v} / \mathrm{v}, 2 \mathrm{~g} / \mathrm{kg})$ or saline once a day for 7 or $14 \mathrm{~d}$. Sixteen or forty hours after the last injection, striatal slices were prepared for electrophysiological recordings, or striatal tissues were dissected for biochemical measurements.

\section{Blood ethanol concentration measurements}

Trunk blood was collected in heparinized capillary tubes $15 \mathrm{~min}$ after systemic administration of $2 \mathrm{~g} / \mathrm{kg}$ of ethanol. Serum was extracted with $3.4 \%$ trichloroacetic acid followed by a 5 min centrifugation at $420 \times g$ $(2000 \mathrm{rpm})$ and assayed for ethanol content using the NAD-NADH enzyme spectrophotometric method (Weiss et al., 1993; Zapata et al., 2006). Blood ethanol concentrations (BECs) were determined by using a standard calibration curve. BEC measurements were performed on 3 Sprague Dawley rats (3-4 weeks old), and the BEC average value was $34 \pm 3 \mathrm{~mm}$.

\section{Electrophysiology}

Slice preparation. The preparation of striatal slices and whole-cell recordings of striatal neurons were done as previously described (Wang et al., 2007). Specifically, Sprague Dawley rats were anesthetized with isoflurane. Coronal sections of the striatum $(150 \mu \mathrm{m})$ were cut with a vibratome (Leica) in an ice-cold solution containing the following (in $\mathrm{mm}$ ): $40 \mathrm{NaCl}, 85$ choline chloride, $4 \mathrm{KCl}, 1.25 \mathrm{NaH}_{2} \mathrm{PO}_{4}, 26 \mathrm{NaHCO}_{3}, 0.5$ $\mathrm{CaCl}_{2}, 7 \mathrm{MgCl}_{2}, 10$ glucose, 1 sodium ascorbate, and 3 sodium pyruvate, saturated with $95 \% \mathrm{O}_{2}$ and $5 \% \mathrm{CO}_{2}$. Slices were then incubated in the same solution at $32^{\circ} \mathrm{C}$ for $45 \mathrm{~min}$ before being transferred to a chamber at room temperature that contained an external solution composed of (in mM): $125 \mathrm{NaCl}, 2.5 \mathrm{KCl}, 2.5 \mathrm{CaCl}_{2}, 1.3 \mathrm{MgCl}_{2}, 1.25 \mathrm{NaH}_{2} \mathrm{PO}_{4}, 25$ $\mathrm{NaHCO}_{3}$, and 10 glucose, saturated with $95 \% \mathrm{O}_{2}$ and $5 \% \mathrm{CO}_{2}$.

The method for preparation of slices from adult Long-Evans rats was the same as above except for the following: (1) rats were anesthetized and intracardially perfused with ice-cold cutting solution as described by Wang et al. (2004); (2) slices were incubated in a cutting and external solution (1:1 in volume) for $45 \mathrm{~min}$ before being transferred into the external solution.

Whole-cell voltage-clamp recordings. NMDAR-mediated EPSCs (NMDAREPSCs) were recorded at $-70 \mathrm{mV}$ and in the presence of $50 \mu \mathrm{M} \mathrm{Mg}{ }^{2+}, 10$ $\mu \mathrm{M}$ NBQX, $100 \mu \mathrm{M}$ picrotoxin, and $200 \mu \mathrm{M}$ sodium orthovanadate in the bath solution (Wang et al., 2007). To measure NMDA-induced currents, NMDA was bath applied for $30 \mathrm{~s}$ and holding currents were measured every $5 \mathrm{~s}$. To generate input-output curves for NMDAR-EPSCs, currents responding to increasing intensities of stimulation (3.6 to $7.2 \mathrm{~V}$ with an increment of $0.9 \mathrm{~V}$ ) were recorded, as previously described (Palop et al., 2007). Slices were superfused at $3-4 \mathrm{ml} / \mathrm{min}$, and cells were visualized using infrared differential interference contrast video microscopy (BX50WI, Olympus).

Recordings were made using a MultiClamp 700A amplifier (Molecular Devices). Electrodes (4-6 M $\Omega$ ) contained the following (in mM): 115 cesium methanesulfonate, 15 HEPES, 0.6 EGTA, 8 TEA-Cl, 4 MgATP, $0.3 \mathrm{NaGTP}$, and $7 \mathrm{Na}_{2} \mathrm{CrPO}_{4}$ (pH 7.2-7.3) with an osmolarity of 270 $280 \mathrm{mOsm}$. Series resistance and input resistance were monitored online. Stimulation of inputs to the striatum was achieved with tungsten bipolar electrodes (FHC) that were placed in the DMS or the DLS and used to stimulate excitatory afferents at $0.05 \mathrm{~Hz}$. Neurons were voltage clamped at $-70 \mathrm{mV}$. Data were filtered at $2 \mathrm{kHz}$, digitized at $5-10 \mathrm{kHz}$, and collected on-line using pClamp 9 (Molecular Devices).

\section{DMS and DLS tissue collection and homogenization}

DMS and DLS tissues were collected as described by Jeanblanc et al. (2009). Specifically, after rats were anesthetized by isoflurane and decapitated, brains were removed and one section per animal was cut between $+1.70 \mathrm{~mm}$ and $+0.5 \mathrm{~mm}$ anterior to bregma. Following removal of the cortex and the ventral striatum $(-6.00 \mathrm{~mm}$ below the brain surface), the dorsal striatum was divided into the DMS and the DLS at $+2.5 \mathrm{~mm}$ from the middle line. DMS and DLS were dissected on a cool $\left(4^{\circ} \mathrm{C}\right)$ tray and immediately homogenized in ice-cold radio immunoprecipitation assay (RIPA) buffer (in mm: 50 Tris-Cl, 5 EDTA, and $120 \mathrm{NaCl}$, and $1 \% \mathrm{NP}-40$, $0.1 \%$ deoxycholate, $0.5 \%$ SDS, and protease and phosphatase inhibitors). Samples were homogenized using a sonic dismembrator. Protein content of samples was determined using the BCA protein assay kit.

\section{Synaptosomal membrane preparation}

Synaptosomal membranes were prepared as described by Carvelli et al. (2002) and Daws et al. (2002). Briefly, immediately after being collected, DMS tissue was homogenized in a glass homogenizer containing $200 \mu \mathrm{l}$ of ice-cold Krebs-sucrose buffer (in mm: $125 \mathrm{NaCl}, 1.2 \mathrm{KCl}, 1.2 \mathrm{MgSO}_{4}$, $1.2 \mathrm{CaCl}_{2}, 22 \mathrm{Na}_{2} \mathrm{CO}_{3}, 1.2 \mathrm{NaH}_{2} \mathrm{PO}_{4}, 10$ glucose, and 320 sucrose, $\mathrm{pH}$ $7.4)$ in the presence of protease and phosphatase inhibitors. The homogenate was centrifuged at $1000 \times g$ for $10 \mathrm{~min}$ at $4^{\circ} \mathrm{C}$ to pellet heavy membranes and debris (P1). The supernatant (S1) was collected and was centrifuged at $16,000 \times g$ at $4^{\circ} \mathrm{C}$ for 20 min to pellet the synaptosomal membrane fraction (P2). P2 was resuspended in $100 \mu \mathrm{l}$ of RIPA buffer. Total protein concentration was determined using the BCA protein assay kit.

\section{Immunoprecipitation}

DMS tissues ( $n=3$ rats per treatment) were homogenized in $1 \times$ immunoprecipitation (IP) buffer (in mM: $150 \mathrm{NaCl}, 10$ Tris-HCl, 1 EDTA, 1 EGTA, and 1\% Triton X-100, pH 7.4) in the presence of protease and phosphatase inhibitor cocktails. DMS homogenates were precleared by incubation with protein $\mathrm{G}$ agarose for $1 \mathrm{~h}$ at $4^{\circ} \mathrm{C}$. Samples were then centrifuged and protein quantity was determined using the BCA protein assay. IPs were performed by combining $5 \mu \mathrm{g}$ of the appropriate antibody with $\sim 1 \mathrm{mg}$ of protein diluted in $1 \times \mathrm{IP}$ buffer to a total volume of $1 \mathrm{ml}$. Following overnight incubation at $4^{\circ} \mathrm{C}$, protein $\mathrm{G}$ agarose was added and the mixture was incubated at $4^{\circ} \mathrm{C}$ for $4 \mathrm{~h}$. The protein $\mathrm{G}$ was washed extensively with $1 \times$ IP buffer and pellets were resuspended in $25 \mu$ l of $2 \times$ Laemmli buffer and incubated at $95^{\circ} \mathrm{C}$ for $10 \mathrm{~min}$. The resulting supernatant was separated by SDS-PAGE using 10\% Bis Tris precast gels for $2 \mathrm{~h}$ at $130 \mathrm{~V}$.

\section{Western blot analysis}

Samples were separated on NuPAGE 4-12\% Bis-Tris gradient gels at 130 $\mathrm{V}$ for $2-3 \mathrm{~h}$ unless stated otherwise. After overnight transfer at $4^{\circ} \mathrm{C}$ onto nitrocellulose membranes, blots were blocked in 5\% milk/TBST before probing with specific antibodies followed by horseradish peroxidaseconjugated secondary antibodies. Blots were visualized using ECL Plus 
and a Storm 860 imagining system (Molecular Dynamics). Results were quantified with the Scion Image-Analysis Software (Scion).

\section{Intermittent access two-bottle choice procedure}

The procedure is similar to the one described by Carnicella et al. (2009). Briefly, Long-Evans rats were given $24 \mathrm{~h}$ concurrent access to 1 bottle of $20 \%$ ethanol in water and 1 bottle of water starting at 11:00 A.M. on Monday, Wednesday, and Friday, with 24 or $48 \mathrm{~h}$ ethanol-deprivation periods in between the ethanol-drinking sessions. The placement (left or right) of each solution was alternated between each session to control for side preference. The water and ethanol bottles were weighed after $24 \mathrm{~h}$ of access. Experiments started after a stable baseline of consumption was achieved (average of 5-6 g/kg/24 h) (supplemental Table 1, available at www.jneurosci.org as supplemental material). The control group had access to two bottles of water throughout the duration of the experiment.

\section{Operant ethanol self-administration after history of high voluntary ethanol consumption}

Long-Evans rats were first trained to voluntary consume high levels of ethanol using the intermittent access $20 \%$ ethanol (v/v) two-bottle choice drinking procedure as described above. After achieving a stable baseline of consumption, rats were trained to self-administer a $20 \%$ ethanol solution in an operant self-administration chamber. The chambers contain two levers: an active lever, for which presses result in delivery of $0.1 \mathrm{ml}$ of the ethanol solution, and an inactive lever, for which responses are counted but no programmed events occur. After $2-3$ nights in the chamber to allow acquisition of lever-press response for ethanol under a fixed ratio 1 (FR1), operant sessions were conducted $5 \mathrm{~d}$ per week, with the schedule requirement increased to FR 3 and the length of session shortened from 60 to $30 \mathrm{~min}$ over the first 2 weeks. Because the level of presses on the inactive lever was extremely low after acquisition of the self-administration procedure ( $<10$ presses), and the activity on this lever was not affected by any of the experimental treatments, this measure was excluded from the figures and the analysis for better clarity. After 1 month of training, surgery was conducted to implant cannulae.

\section{Operant self-administration test in extinction and reinstatement} of ethanol seeking

The following behavioral manipulations began after acquisition of a stable operant responding for ethanol as described above.

Test in extinction. Rats experienced a single $30 \mathrm{~min}$ session of extinction per week in which responses on the ethanol lever were counted but no ethanol was delivered.

Reinstatement of ethanol seeking. Rats underwent a minimum of 9 extinction sessions until the average of active lever presses across 3 consecutive extinction sessions was lower than 10. The following session, reinstatement of ethanol seeking, was induced by the noncontingent delivery of $0.2 \mathrm{ml}$ of $20 \%$ ethanol into the reward port immediately at the beginning of the session, followed by an additional $0.1 \mathrm{ml}$ of $20 \%$ ethanol after the completion of the first 3 presses on the ethanol lever. During the remainder of the $30 \mathrm{~min}$ session, subsequent responses on the active lever were counted but no ethanol was delivered. This oral ethanol priming is not sufficient to produce a pharmacological effect (intake $<0.1 \mathrm{~g} / \mathrm{kg}$ ); however, it enables access to the exteroceptive properties of ethanol (i.e., taste and odor) and has been shown to reinstate lever pressing on the ethanol lever (Carnicella et al., 2008; Chaudhri et al., 2008). The experiment was conducted by using a counterbalanced within-subjects design (resulting in 3 tests per subject) and as such, reinstatement tests were followed by 1 week of reacquisition of ethanol self-administration and then further extinction sessions before the next reinstatement test (Carnicella et al., 2008). This procedure of reacquisition between 2 extinction and reinstatement procedures prevents any decrease in the efficacy of the prime due to repeated exposure, as the level of reinstatement for the vehicle condition was similar during the 3 tests (data not shown).

\section{Operant sucrose self-administration}

Long-Evans rats were initially trained under FR1 using $8 \%$ sucrose (w/v) during 2 overnight sessions. The FR schedule was then progressively increased to FR3, and the sucrose concentration was progressively decreased to $2.5 \%$, while the length of the session was shortened to $30 \mathrm{~min}$.
These parameters were chosen to obtain a lever-press activity similar to that observed in operant ethanol self-administration.

\section{Surgery and microinjection of PP2 and ifenprodil}

Long-Evans rats were anesthetized continuously with isoflurane (Baxter Health Care). Bilateral guide cannulae (C235G, 26 ga, Plastics One) were implanted into the DMS ( $1.2 \mathrm{~mm}$ anterior to bregma, $1.5 \mathrm{~mm}$ mediolateral, $4.2 \mathrm{~mm}$ ventral to bregma) or into the DLS (1.2 $\mathrm{mm}$ anterior to bregma, $3.4 \mathrm{~mm}$ mediolateral, $4.2 \mathrm{~mm}$ ventral to bregma), according to Paxinos and Watson (2007), and were based on Corbit and Janak (2007). Drug or vehicle was administered via an injection cannulae extending 0.5 $\mathrm{mm}$ beyond the guide cannula tip. The volume infused with a Hamilton $25 \mu \mathrm{l}$ syringe (\# 1702) was $1 \mu \mathrm{l} /$ side at a rate of $0.5 \mu \mathrm{l} / \mathrm{min}$, with the injector kept in position for an additional $2 \mathrm{~min}$. All subjects received each drug in a counterbalanced fashion, with at least 1 week between 2 infusions. The doses used were 0.3 and $1.5 \mathrm{ng} / \mu \mathrm{l}$ for PP2, and 0.5 and 2.5 $\mu \mathrm{g} / \mu \mathrm{l}$ for ifenprodil. PP2 and ifenprodil were initially dissolved in DMSO and brought to a final concentration of $1 \%$ DMSO with PBS. Doses and time of infusion of PP2 and ifenprodil were based on Wang et al. (2007).

\section{Histology}

Locations of cannulae were verified in $60 \mu \mathrm{m}$ coronal sections of paraformaldehyde-fixed tissue stained with thionin. Only data from subjects with cannulae located in the region of interest were included in the analysis (supplemental Fig. 1, available at www.jneurosci.org as supplemental material).

\section{Statistical analysis}

All data are expressed as mean \pm SEM unless stated otherwise.

Statistical analyses for electrophysiological data were performed using two-way ANOVA for repeated measures, followed by the Student-Newman-Keuls (SNK) post hoc test (two-way RM-ANOVA) (Figs. $1 A, 2 B, D$, $6 B, D$ ) or using the unpaired, two-tailed $t$ test (Figs. $2 A, C, 3,6 A, C$ ). Specifically in Figure $1 A$, the brain region (DMS and DLS) was analyzed as a between-subject factor and the ethanol treatment as a within-subject factor, which consists of three time periods: baseline, EtOH (during ethanol application), and post-EtOH (30 min after ethanol washout), which correspond to the time periods $1-3$ in Figure $1 A b$, respectively. At each time period, 10 min EPSCs were averaged to construct one data point for each subject. To closely examine the interaction between the two factors, only two time periods (EtOH and post-EtOH) were analyzed. In Figures $2 B, 2 D, 6 B$, and $6 D$, the ethanol treatment was analyzed as a betweensubject factor and the stimulation intensity as a within-subject factor, which consists of 5 different stimulation intensities (from 3.6 to $7.2 \mathrm{~V}$ with an increment of $0.9 \mathrm{~V}$ ).

For the biochemical analyses, the ratio of the intensity of the immunoreactive band of interest to GAPDH was determined for each sample to normalize for the amount of protein loaded, and the resultant data were analyzed using unpaired, two-tailed $t$ test. When data did not conform to a normal distribution, the analyses were conducted using the Mann-Whitney rank sum test.

Behavioral data were analyzed using a one-way ANOVA with repeated measures (one-way RM-ANOVA), followed by SNK test when indicated. As the latency data to the third press (i.e., first reward) did not conform to a normal distribution, the analyses were conducted using the Friedman repeated-measures ANOVA on ranks.

\section{Results}

\section{Acute ethanol exposure and withdrawal upregulate the NMDAR activity in the DMS}

We first determined whether acute exposure of striatal slices to, and withdrawal from, ethanol differentially alters the activity of NMDARs in the DMS and the DLS. After a stable recording of NMDAR-mediated EPSCs for $10 \mathrm{~min}$, ethanol (40 mM) was bath applied for $15 \mathrm{~min}$, then washed out (withdrawal) for another 30 min, and the EPSCs were continuously measured. As shown in Figure $1 A$, the NMDAR-EPSCs were reduced in the presence of ethanol ( $p<0.001$ vs baseline in the DMS and DLS), and the 
A a

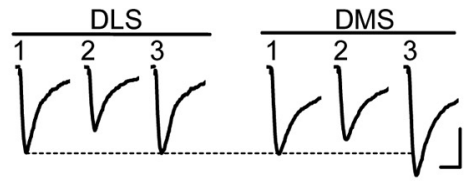

b

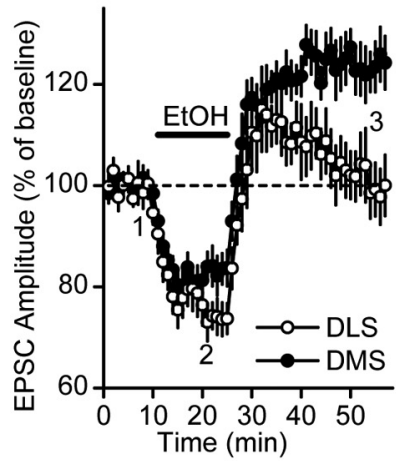

C

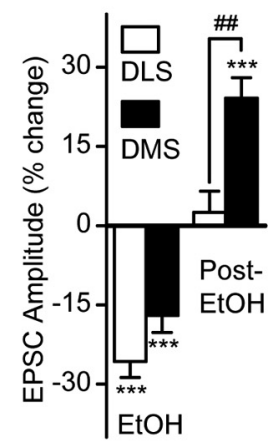

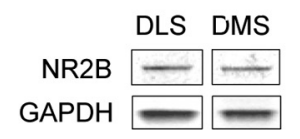
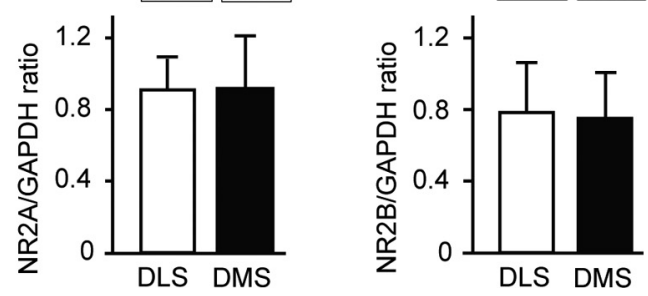

Figure 1. Long-term facilitation of NMDAR-mediated EPSCs is observed in the DMS but not in the DLS. Aa, Sample traces of DLS (left) and DMS (right) EPSCs at time periods $1-3$ that are indicated in $\boldsymbol{A} \boldsymbol{b}$. The stimulus artifacts have been omitted for clarity. Calibration: $50 \mathrm{~ms}, 50 \mathrm{pA}$. $\boldsymbol{A} \boldsymbol{b}$, Time course of NMDAR-mediated EPSCs before, during (15 min, as indicated by the horizontal line), and after ethanol (40 mM) application. $n=12$ (DLS) and 10 (DMS) slices. Ac, Bar graph comparing the direct (EtOH) and washout (post-EtOH) effects of ethanol on NMDARmediated EPSCS. EPSCS were averaged from 5-14 min after ethanol application (EtOH) and from 21-30 min after ethanol washout (post-Et0H). ${ }^{* * *} p<0.001$ versus baseline; ${ }^{* \#} p<0.01$ DMS versus DLS. Two-way RM-ANOVA with SNK post hoc tests was used. $B$, NR2A and NR2B protein levels in the DMS and DLS. Data are shown as ratio of NR2A or NR2B to GAPDH \pm SD, $n=9$ for each subregion. $p>0.05$ DMS versus DLS (two-tailed $t$ test).

amplitude of NMDAR-mediated EPSCs was greatly enhanced immediately after ethanol withdrawal in both subregions. However, the EPSCs remained elevated in the DMS $(p<0.001$ vs baseline), but not in the DLS ( $p=0.48$ vs baseline) for $30 \mathrm{~min}$ after ethanol withdrawal (Fig. $1 A$ ). The analysis of NMDAREPSCs by two-way RM-ANOVA further reveals a significant interaction between the brain region and the ethanol treatment $\left(F_{(1,20)}=4.47, p<0.05\right.$, see Materials and Methods). Post hoc comparison by SNK tests reveals that NMDAR-EPSC amplitudes are different between the DMS and the DLS 30 min after ethanol washout (post-EtOH, $p<0.01$ ), but not during ethanol application $(\mathrm{EtOH}, p=0.29)$. This result therefore suggests that exposure of striatal slices to, and withdrawal from, ethanol induces LTF of NMDAR activity in the DMS, but not in the DLS.

Next, we tested whether the differential effects of ethanol exposure on the activity of the NMDAR in the subregions of the dorsal striatum are due to different protein levels of the NR2A and/or the NR2B subunits, the major regulatory NR2 subunits in the dorsal striatum (Monyer et al., 1992; Standaert et al., 1994). As shown in Figure $1 B$, we did not observe any differences between the protein levels of NR2A or NR2B in the DMS and the $\operatorname{DLS}\left(t_{(16)}=0.05, p=0.96\right.$ for NR2A and $t_{(16)}=0.39, p=0.71$ for

NR2B). Furthermore, the degree of inhibition of NMDARmediated EPSCs in the presence of an NR2B-NMDAR-specific antagonist, Ro 25-6981, was similar in the DMS and DLS of naive rats (data not shown), suggesting that the level of synaptic responses are similar in the two subregions.

\section{Repeated ethanol administration increases NMDA activity in the DMS}

Next, we tested whether in vivo exposure to, and withdrawal from, ethanol results in changes in the activity of NMDARs in the DMS. To correlate the results obtained ex vivo described above with an in vivo procedure, we chose to systemically administer a dose of $2 \mathrm{~g} / \mathrm{kg}$, which results in a peak BEC of $34 \pm 3 \mathrm{~mm}$ (see Materials and Methods) that is similar to the concentration of ethanol that induces LTF of NMDARs in striatal slices (Fig. 1A). Rats were given a single systemic injection of ethanol or saline and NMDA-induced currents were measured in DMS neurons $16 \mathrm{~h}$ later. A single systemic injection of ethanol did not result in a detectable alteration of NMDAR activity in the DMS (data not shown). We therefore hypothesized that prolonged exposure to, and withdrawal from, ethanol leads to a gradual long-lasting increase in the activity of the channel in the DMS (see diagram, Fig. 10). To model this possibility, rats received a daily systemic administration of ethanol ( $2 \mathrm{~g} / \mathrm{kg}$, i.p.) or saline for $7 \mathrm{~d}$, and the activity of the channel was measured $16 \mathrm{~h}$ after the seventh injection. We found that daily repeated administration of ethanol produced a greater NMDA-elicited change in holding currents in DMS neurons than in saline-treated rats (Fig. $2 A)\left(t_{(26)}=-3.02\right.$, $p<0.01)$. Since the NMDA current was elicited by bath application of NMDA, which activates both synaptic and extrasynaptic NMDARs (Carpenter-Hyland et al., 2004), the differences in the NMDA-induced currents between saline and ethanol treatments may result from changes in synaptic and/or extrasynaptic NMDARs. To address this question, we measured the inputoutput relation for NMDAR-EPSCs in DMS neurons from rats that received saline or ethanol treatments. As shown in Figure $2 B$, EPSCs are higher in DMS neurons from ethanol- than from saline-treated animals (main effect of stimulation intensity: $F_{(4,146)}=30.54, p<0.001$; main effect of ethanol treatment: $F_{(1,146)}=5.47, p<0.05$; interaction between both factors: $F_{(4,146)}=$ $3.44, p=0.01)$, suggesting that repeated ethanol administration induces an increase in the activity of synaptic NMDARs in the DMS. Interestingly, the frequency of miniature EPSCs (mEPSCs) was not altered $16 \mathrm{~h}$ after the last administration of ethanol (data not shown), suggesting that the changes in NMDAR activity in response to ethanol exposure and withdrawal are postsynaptic.

Seven daily treatments of rats with ethanol did not produce a change in the activity of the NMDAR in the DMS $40 \mathrm{~h}$ after the last administration (data not shown). We therefore tested whether there is a correlation between the duration of the repeated ethanol exposure and withdrawal, and the extent of enhancement of NMDAR activity. Rats were treated daily with 2 $\mathrm{g} / \mathrm{kg}$ ethanol or saline for $14 \mathrm{~d}$ and the activity of the channel was determined $40 \mathrm{~h}$ after the last injection. We observed a significantly higher amplitude of NMDA-induced currents in the DMS of ethanol-treated animals than in saline-treated ones (Fig. $2 C$ ) $\left(t_{(49)}=-3.72, p<0.001\right)$, and a higher amplitude of NMDAREPSCs (Fig. 2D) (main effect of stimulation intensity: $F_{(4,92)}=$ 69.06, $p<0.001$; main effect of ethanol treatment: $F_{(1,92)}=10.22$, $p<0.01$; interaction between both factors: $F_{(4,92)}=8.89, p<$ $0.001)$. Together, our results suggest that repeated exposure of 
A

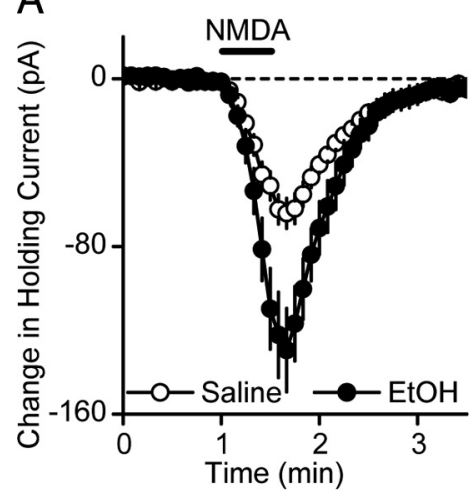

C

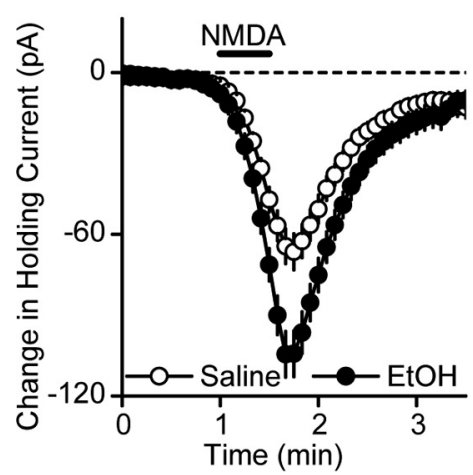

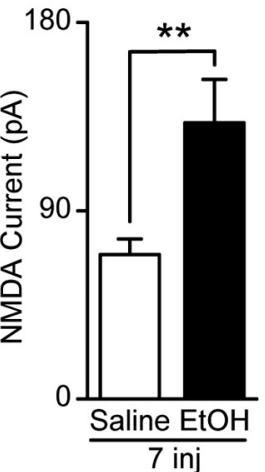

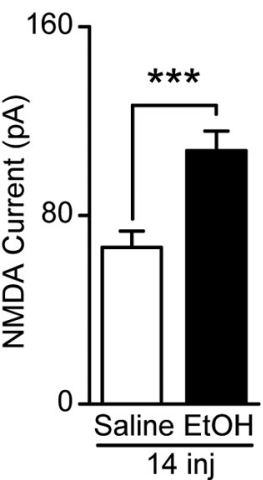

B
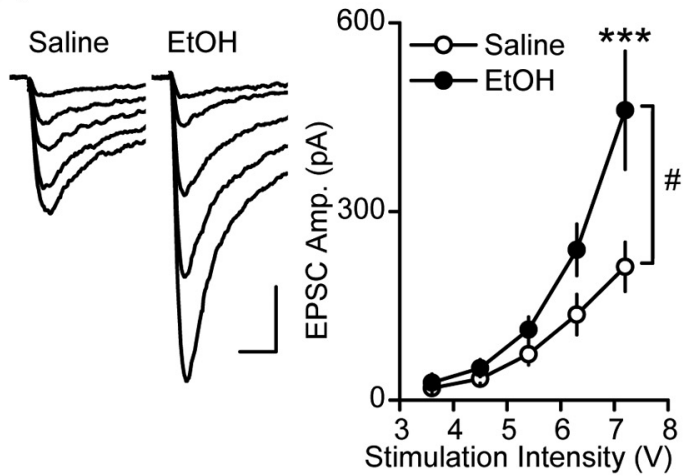

$\mathrm{D}$

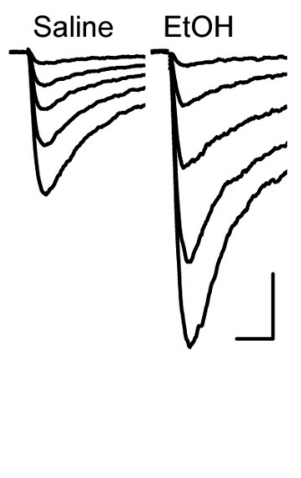

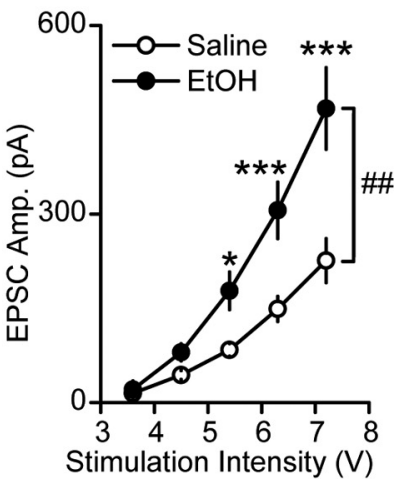

Figure 2. Repeated ethanol administration leads to an increase in NMDAR activity in the DMS. $\boldsymbol{A}, \boldsymbol{B}$, Repeated administration of ethanol leads to increases in NMDA-induced currents ( $\boldsymbol{A}$ ) and synaptic NMDAR-EPSCS $(\boldsymbol{B})$ in the DMS, $16 \mathrm{~h}$ after the last treatment. Rats received systemic administration of ethanol $(2 \mathrm{~g} / \mathrm{kg}$, i.p.) or saline once a day for $7 \mathrm{~d}$. $\boldsymbol{A}$, Left, Changes in holding currents were measured after NMDA $(10 \mu \mathrm{m}, 30 \mathrm{~s})$ was applied to the slices. $n=15$ (saline) and 13 (EtOH). Right, Bar graph summarizing the NMDA-induced peak currents in DMS neurons from ethanol-and saline-treated rats. ${ }^{* *} p<0.01$ by $t$ test. $B$, Left, Representative traces of NMDAR-EPSCs evoked by a range of stimulation intensity in slices from saline- and ethanol-treated rats. Calibration: $40 \mathrm{~ms}$, 100 pA. Right, Input- output curves for NMDAR-EPSCs in DMS neurons from saline- and ethanol-treated rats. $n=20$ (saline) and 21 (EtOH). ${ }^{\#} p<0.05 .{ }^{* * *} p<0.001$ versus saline at the same stimulation intensity. Two-way RM-ANOVA with SNK post hoc tests. C, $\boldsymbol{D}$, Repeated administration of ethanol for $14 \mathrm{~d}$ leads to increases in NMDA-induced currents ( $(\boldsymbol{C})$ and synaptic NMDAR-EPSCS (D) $40 \mathrm{~h}$ after the last treatment. Rats received systemic administration of ethanol $(2 \mathrm{~g} / \mathrm{kg}$, i.p.) or saline once a day for $14 \mathrm{~d}$, and the function of NMDARs was tested in the DMS $40 \mathrm{~h}$ after the last injection. C, Left, Changes in holding currents were measured after NMDA (10 $\mu \mathrm{M}, 30 \mathrm{~s}$ ) was applied to the slices. $n=24$ (saline) and 27 (EtOH). Right, Bar graph summarizing the NMDA-induced peak currents in DMS neurons from ethanol- and saline-treated rats. ${ }^{* * *} p<0.001$ by $t$ test. $D$, Left, Representative traces of NMDAR-EPSCs evoked by a range of stimulation intensities in slices from saline- and ethanol-treated rats. Calibration: 40 ms, 100 pA. Right, Input- output curves for NMDAR-EPSCs in DMS neurons from saline- and ethanol-treated rats. $n=12$ (saline) and 13 (EtOH) $\# p<0.01 .{ }^{*} p<0.05$ and ${ }^{* * *} p<0.001$ versus saline at the same stimulation intensity. Two-way RM-ANOVA with SNK post hoc tests.

rats to ethanol results in a progressive and long-lasting upregulation of the activity of NMDARs in the DMS.

Repeated administration of ethanol leads to a long-lasting increase in the contribution of NR2B subunits to the activity of the channel in the DMS

Next, we tested for the contribution of the NR2B subunit to the long-lasting upregulation of the activity of the channel after daily exposure to ethanol. Rats were treated with ethanol $(2 \mathrm{~g} / \mathrm{kg}$, i.p., once daily for $7 \mathrm{~d}$ ) or saline, slices were prepared $16 \mathrm{~h}$ after the last injection, and the activity of the channel in the DMS was determined in presence of Ro 25-6981. As shown in Figure 3, a greater inhibition of the NR2B-containing NMDAR-mediated synaptic transmission was observed in the DMS of ethanol-treated rats as opposed to the saline-treated ones $\left(t_{(30)}=-2.63, p<0.05\right)$, suggesting that repeated in vivo exposure to, and withdrawal from, ethanol results in a long-lasting increase in the contribution of NR2B subunits to the overall activity of the channel.

Repeated ethanol administration leads to increased phosphorylation of synaptosomal NR2B in the DMS A potential mechanism to account for the prolonged upregulation of NR2B-NMDAR activity in response to ethanol exposure
$A$

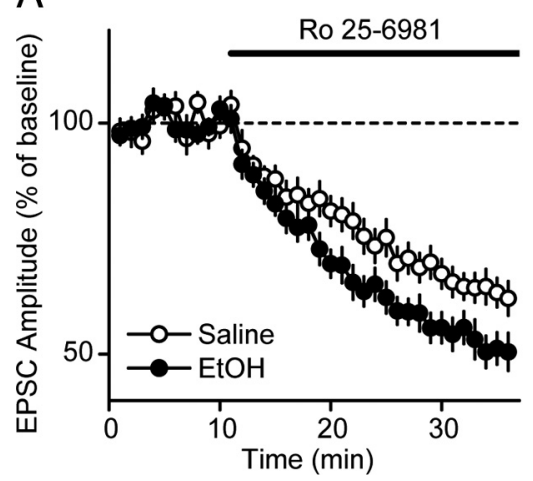

B

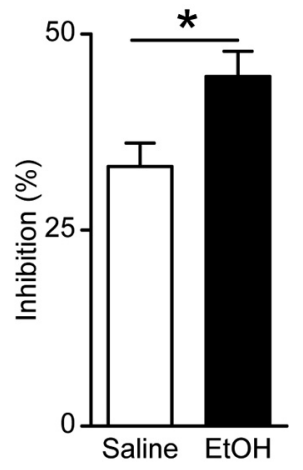

Figure 3. Repeated ethanol administration results in an increase in contribution of NR2B subunits to the activity of the channel in the DMS. $A$, Time course of NMDAR-EPSCs in DMS neurons from ethanol- and saline-treated rats before and during bath application of the NR2BNMDAR-specific antagonist, Ro 25-6981 (0.25 $\mu \mathrm{m}$ ) (as indicated by the horizontal line). Rats received injections of ethanol ( $2 \mathrm{~g} / \mathrm{kg}$, i.p.) or saline once a day for $7 \mathrm{~d}$. DMS slices were prepared $16 \mathrm{~h}$ after the last injection. $n=17$ (saline) and 15 (EtOH) slices. $\boldsymbol{B}$, Summary of the mean inhibition magnitude of NMDAR-EPSCs by Ro 25-6981 in DMS neurons from saline- and ethanol-treated animals. ${ }^{*} p<0.05, t$ test. 
and withdrawal is an increase in the forward trafficking of the NR2B-containing NMDARs to the membrane (Qiang et al., 2007). To address this possibility, we isolated synaptosomes from the DMS of rats $16 \mathrm{~h}$ after the seventh injection of saline or ethanol and measured the level of synaptosomal NR2B subunit in the DMS. As shown in Figure $4 A$, although the protein level of the NR2B subunit in the synaptosomal fraction was only marginally increased after repeated administration of ethanol and withdrawal (two-tailed $t$ test: $\left.t_{(10)}=-1.92, p=0.08\right)$, the levels of NR1 or NR2A in synaptosomal fractions (data not shown), as well as the total protein level of NR2B (Fig. $4 B)\left(t_{(16)}=0.31, p=\right.$ $0.76)$, NR1 and NR2A subunits were unaltered (data not shown), suggesting a specific increase of the NR2B subunit in membranes. Tyrosine phosphorylation of the NR2B subunit on residue Y1472 has been linked to the forward trafficking of the NR2B-NMDARs from intracellular compartments to the synaptic membrane (Dunah and Standaert, 2001; Hallett et al., 2006). Tyrosine phosphorylation of Y1472 was also shown to stabilize the subunit at the membrane by preventing endocytosis of the receptor (Prybylowski et al., 2005; Hayashi et al., 2009). In addition, we previously showed that acute ex vivo exposure of hippocampal or striatal slices to ethanol leads to increased tyrosine phosphorylation of the NR2B subunit in total homogenates (Yaka et al., 2003; Wang et al., 2007), and that acute systemic administration of ethanol leads to an increase in the phosphorylation state of the NR2B subunit in the dorsal striatum (Wang et al., 2007). We therefore tested whether the NR2B subunit in the synaptosomal fraction was tyrosine phosphorylated $16 \mathrm{~h}$ after the seventh administration of ethanol or saline. As shown in Figure $4 A$, repeated ethanol exposure resulted in a striking long-lasting increase in the phosphorylation level of synaptosomal NR2B subunits $\left(t_{(10)}=-4.05, p<0.01\right)$.

Together, these results suggest that repeated in vivo exposure to ethanol causes a long-term increase in the level and the phosphorylation state of the NR2B subunit in synaptic membranes of the DMS, and in increased function of the NR2B-NMDARs.

\section{Repeated administration of ethanol and withdrawal leads to long-lasting activation of Fyn kinase in the DMS}

We, and others, previously showed that Fyn phosphorylates the NR2B subunit of the NMDAR, which results in an increase in channel function (Yaka et al., 2002, 2003; Prybylowski et al., 2005; Hayashi et al., 2009). Furthermore, we found that acute ex vivo and in vivo exposure of the dorsal striatum to ethanol leads to increased activity of Fyn (Wang et al., 2007). We also showed that
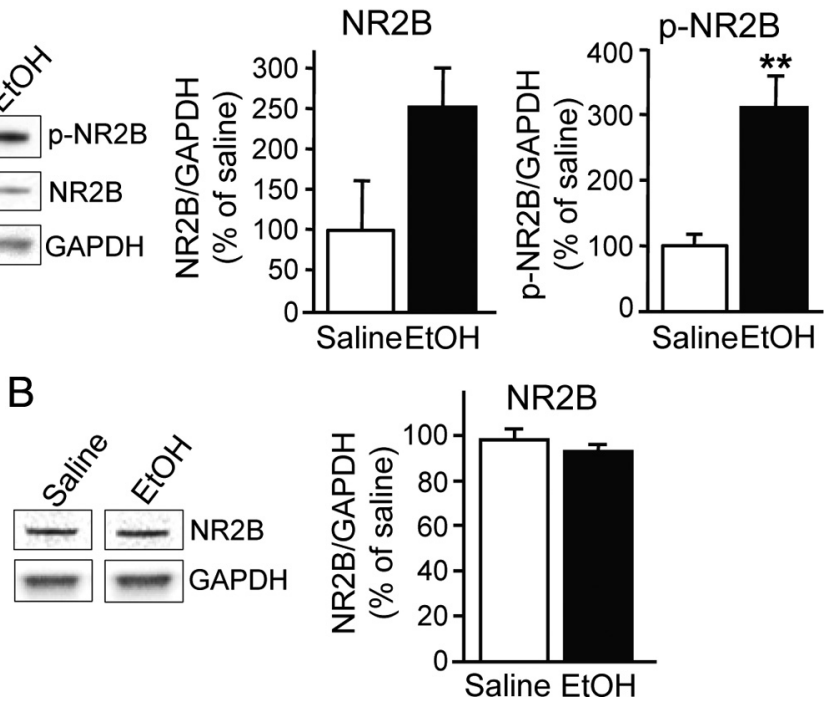

Figure 4. Repeated administration of ethanol increases the phosphorylation level of the NR2B subunit in the synaptosomal membranes of the DMS. Rats were treated with saline or ethanol $(2 \mathrm{~g} / \mathrm{kg}$, i.p.) once a day for $7 \mathrm{~d}$. The DMS was dissected $16 \mathrm{~h}$ after the last injection. $\boldsymbol{A}$, Repeated ethanol exposure increased the phosphorylation level of synaptosomal NR2B subunits. Phospho(p-NR2B) and anti-NR2B antibodies were used to detect the phosphorylated form and the total amount of the NR2B subunit (he averaged changes in the protein (middle) and phosphorylation levels (right) of synaptosomal NR2B 作 wo-tailed $t$ test. $\boldsymbol{B}$, Repeated ethanol administration does not alter the protein levels of NR2B subunits in total DMS homogenates.
A

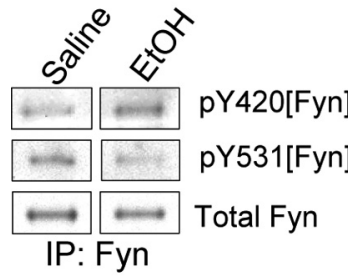

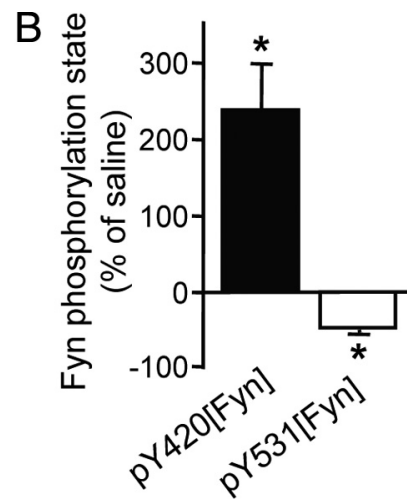

Figure 5. Repeated administration of ethanol increases Fyn activation in the DMS. Rats were treated with ethanol ( $2 \mathrm{~g} / \mathrm{kg}$, i.p.) or saline once a day for $7 \mathrm{~d}$. DMS tissue was dissected out $16 \mathrm{~h}$ after the last treatment. Fyn kinase in total homogenates was immunoprecipitated with anti-Fyn antibodies ( $\boldsymbol{A}$, left panel) and anti-mouse lg $\mathrm{G}$ antibodies as a control $(\boldsymbol{A}$, right panel). Active Fyn was detected by the anti-pY420/418[Fyn/Src] antibodies. The blot was stripped and reprobed first with antibodies that recognize the inactive form of the kinase (anti-pY531/529[Fyn/Src] antibodies), and second with anti-Fyn antibodies (to measure the level of immunoprecipitated Fyn). Images are representative of $n=12$ for each treatment. $\boldsymbol{B}$, Bar graph summarizing the averaged effect of ethanol treatment on the levels of the phosphorylation state of Fyn by quantification of the level of pY420/418 or pY531/529 to total immunoprecipitated Fyn. ${ }^{*} p<0.05$ versus saline (Mann-Whitney rank sum test).

Fyn is required for the acute ethanol-mediated LTF in the dorsal striatum (Wang et al., 2007). Therefore, we were interested in determining whether the observed long-lasting increase in NR2B subunit phosphorylation induced by repeated ethanol exposure correlates with an increase in the activity of Fyn in the DMS. To do so, total homogenates were prepared from the DMS of rats $16 \mathrm{~h}$ after the seventh injection of saline or ethanol. Fyn was immunoprecipitated, and Fyn activity was detected using phospho-specific antibodies that recognize the active (pY420[Fyn]) or inactive (pY531[Fyn]) form of the kinase. We found that the levels of active kinase were elevated (Mann-Whitney rank sum test, $p=0.02$ ), whereas the levels of inactive Fyn were decreased 
A
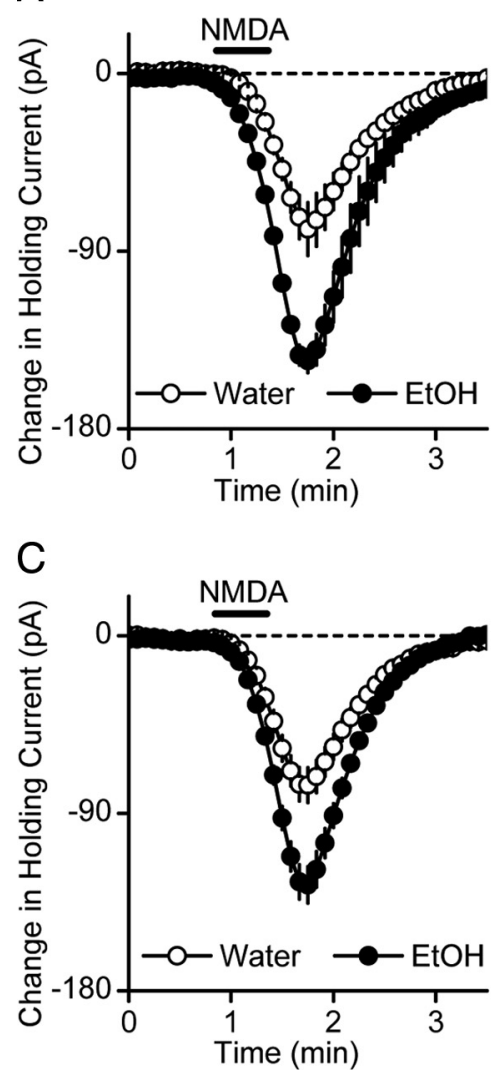
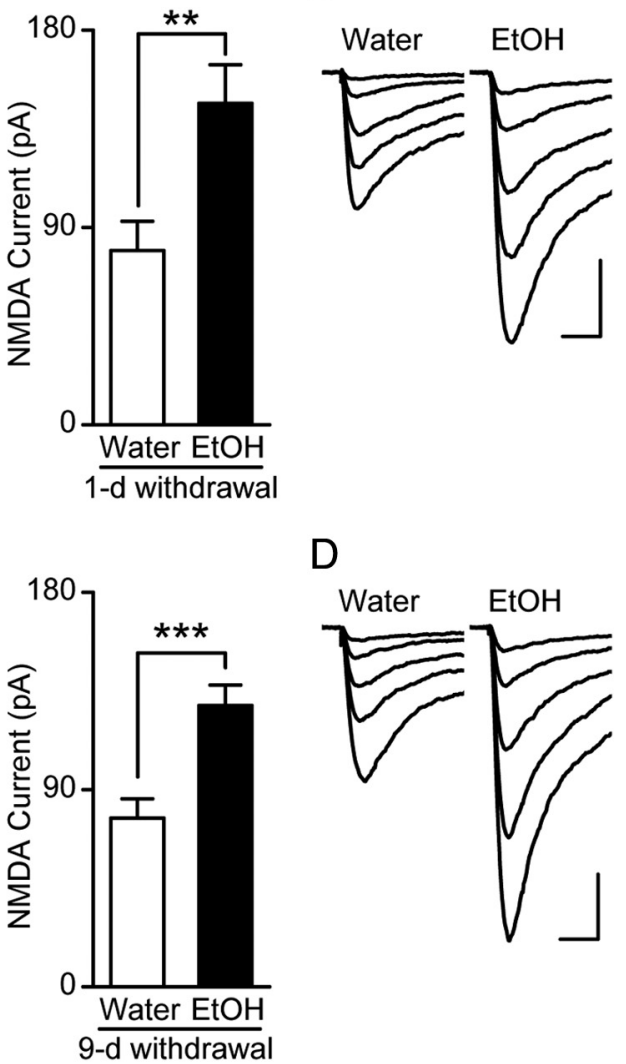

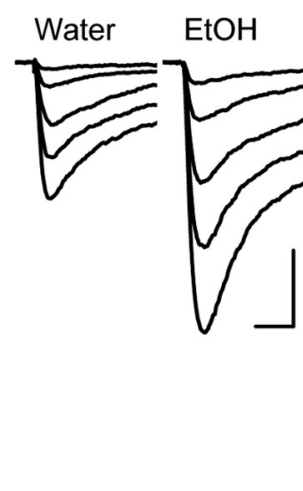

$\mathrm{D}$
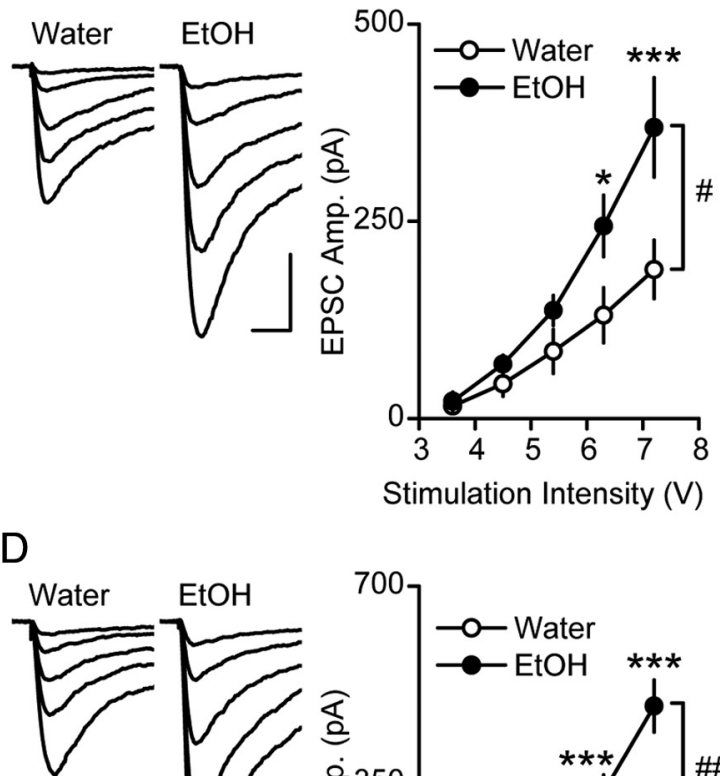

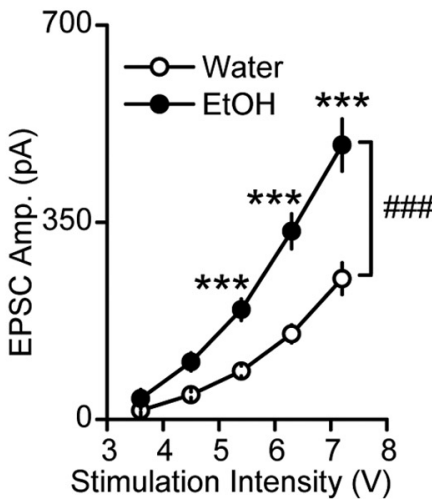

Figure 6. Intermittent access to ethanol results in an increase in NMDAR activity in the DMS. $\boldsymbol{A}, \boldsymbol{B}$, High level of voluntary ethanol consumption leads to increases in NMDA-induced currents ( $\boldsymbol{A}$ ) and synaptic NMDAR-EPSCS $(\boldsymbol{B})$ in the DMS $1 \mathrm{~d}$ after the last ethanol-drinking session. Rats were trained to voluntarily consume a high level of ethanol in an intermittent access to $20 \%$ ethanol two-bottle choice drinking procedure, and rats consuming water only were used as controls. One day after the last drinking session, slices were prepared and DMS neurons were recorded. $A$, Left, Changes in holding currents were measured after NMDA $(15 \mu \mathrm{m}, 30 \mathrm{~s}$ ) was applied to the slices. $n=18$ (water) and 19 (EtOH). Right, Bar graph summarizing the NMDA-induced peak currents in DMS neurons from ethanol-drinking rats and rats that consumed water only. ${ }^{* *} p<0.01, t$ test. $\boldsymbol{B}$, Left, Representative traces of NMDAR-EPSCs evoked by a range of stimulation intensity in slices from ethanol-drinking rats and water controls. Calibration: $40 \mathrm{~ms}, 100 \mathrm{pA}$. Right, Input- output curves of NMDAR-EPSCs in DMS neurons from ethanol and water groups of rats. $n=19$ (water) and 15 (EtOH). ${ }^{\#} p<0.05 .{ }^{*} p<0.05$ and ${ }^{* * *} p<0.001$ versus water at the same stimulation intensity. Two-way RM-ANOVA with SNK post hoc tests. $\boldsymbol{C}, \boldsymbol{D}$, Excessive ethanol consumption causes increases in NMDA-induced currents $(\boldsymbol{C})$ and synaptic NMDAR-EPSCS $(\boldsymbol{D})$ in the DMS, $9 \mathrm{~d}$ after the last ethanol-drinking session. $\boldsymbol{C}, n=32$ (water) and 30 (EtOH). ${ }^{* * *} p<0.001$ by $t$ test. $\boldsymbol{D}, n=27$ for each group. ${ }^{\# \#} p<0.001$. ${ }^{* * *} p<0.001$ versus water at the same stimulation intensity by two-way RM-ANOVA with SNK post hoc tests.

(Mann-Whitney rank sum test, $p=0.02$ ), in the DMS of ethanoltreated rats compared to saline-treated animals (Fig. 5). Together these data suggest that repeated daily exposure to ethanol results in long-lasting robust activation of Fyn kinase, which could account for the sustained upregulation of NR2B phosphorylation and the increased synaptosomal localization of the subunit.

\section{High levels of ethanol consumption result in increased} NMDAR activity in the DMS of adult rats

We reasoned that the daily systemic exposure of rats to ethanol resembles the intermittent access two-bottle choice procedure in which rats have access to a high concentration of ethanol (20\%) every other day (Wise, 1973), We previously showed that this procedure results in excessive level of ethanol consumption $(1-2 \mathrm{~g} / \mathrm{kg}$ in $30 \mathrm{~min})$, leading to an average BEC of $\sim 20-30$ mM (Carnicella et al., 2009), which corresponds to BEC induced by binge drinking in humans (National Institute on Alcohol Abuse and Alcoholism, 2004). We therefore examined whether the activity of the NMDAR in the DMS is enhanced as a result of the repeated cycles of ethanol exposure and withdrawal. Rats underwent an intermittent access to $20 \%$ ethanol in a two-bottle choice drinking procedure as described in Materials and Methods. After achieving a baseline of high levels of ethanol consump- tion (supplemental Table 1, available at www.jneurosci.org as supplemental material), NMDAR activity was measured in the DMS $1 \mathrm{~d}$ after the last ethanol-drinking session, and compared to the activity of the channel in the DMS from a group of rats that were consuming only water. We found that excessive ethanol consumption induces higher amplitude of NMDA-induced currents in the DMS than in water controls (Fig. 6A) $t_{(35)}=-3.02$, $p<0.01$ ) and of NMDAR-EPSCs (Fig. $6 B$ ) (main effect of stimulation intensity: $F_{(4,128)}=55.41, p<0.001$; main effect of ethanol treatment: $F_{(1,128)}=4.57, p<0.05$; interaction between both factors: $\left.F_{(4,128)}=6.50, p<0.001\right)$. These results suggest that repeated cycles of excessive ethanol intake cause an upregulation in the activity of NMDARs in the DMS, at least in part, at the synaptic site.

To determine whether the increase in NMDAR activity is long-lasting, rats underwent the same voluntary ethanol intake procedure and the activity of NMDARs was measured $9 \mathrm{~d}$ after the last ethanol consumption session. We observed that the NMDA-induced current in the DMS was still higher after $9 \mathrm{~d}$ of ethanol deprivation than in rats drinking water only (Fig. $6 C$ ) $\left(t_{(60)}=-3.98, p<0.001\right)$. The amplitude of NMDAR-EPSCs was also higher in the ethanol group than in water group (Fig. $6 D$ ) (main effect of stimulation intensity: $F_{(4,208)}=149.57, p<$ 
0.001; main effect of ethanol treatment: $F_{(1,208)}=27.13, p<$ 0.001 ; interaction between effects of ethanol treatment and stimulation intensity: $\left.F_{(4,208)}=15.44, p<0.001\right)$. These results suggest that repeated cycles of voluntary ethanol intake and deprivation cause a persistent increase in the activity of synaptic NMDARs in the DMS.

High levels of ethanol consumption lead to an increase in the phosphorylation level of NR2B in the DMS

Next we determined whether the phosphorylation level of the NR2B subunit is elevated after repeated excessive ethanol drinking and withdrawal. Rats experienced an intermittent access to $20 \%$ ethanol two-bottle choice procedure as above, and the level of NR2B phosphorylation was measured $24 \mathrm{~h}$ after the end of the last ethanol-drinking session (i.e., $24 \mathrm{~h}$ withdrawal). As shown in Figure $7 A$, the phosphorylation level of NR2B was markedly increased in the DMS of rats consuming ethanol as compared to rats that consumed only water $\left(t_{(13)}=-2.67, p<0.05\right)$, suggesting that ethanol drinking results in a long-lasting increase in NR2B-NMDAR activity. In addition, similar to what we observed using the repeated systemic administration of ethanol (Fig. 4B), the increase in the phosphorylation levels of NR2B did not correspond to increases in total protein level of the NR2B subunit (Fig. $7 B)\left(t_{(13)}=0.19, p=0.85\right), \mathrm{NR} 1$, or NR2A (data not shown).

\section{Inhibition of Src PTKs or NR2B-NMDARs}

in the DMS, but not in the DLS, significantly decreases operant ethanol self-administration in rats Finally, we investigated the possible behavioral consequences of the long-lasting increase in NR2B-NMDAR function in the DMS induced by repeated ethanol exposure. We therefore tested whether inhibition of NR2B-NMDARs and/or Fyn activity in the DMS alters operant ethanol self-administration in rats following a history of high voluntary ethanol consumption. We found that intra-DMS infusion of the NR2B-NMDAR inhibitor, ifenprodil, or the Src PTKs inhibitor, PP2 (which inhibits Fyn), significantly reduced operant responding for ethanol (Fig. 8A) (significant effect of treatment: $\left.F_{(4,20)}=2.91, p<0.05\right)$ and, therefore, ethanol intake (Fig. $8 B$ ) (significant effect of treatment: $\left.F_{(4,20)}=2.98, p<0.05\right)$. Next, we determined the specificity of ifenprodil and PP2 actions in the DMS by examining their ability to attenuate operant self-administration of the natural, non-drug reinforcer, sucrose. Intra-DMS infusion of ifenprodil or PP2 did not alter operant responding for sucrose or sucrose intake [Figure $8 C$ (no significant effect of treatment: $\left.F_{(4,28)}=0.43, p=0.78\right)$ and Figure $8 D$ (no significant effect of treatment: $\left.F_{(4,28)}=0.48, p=0.75\right)$ ], indicating that the decrease in ethanol self-administration induced by NR2B-NMDAR or Src PTK inhibition in the DMS is not due to a general decrease in motivation or to locomotor activity impairment. Importantly, similar treatment into the DLS did not signif-
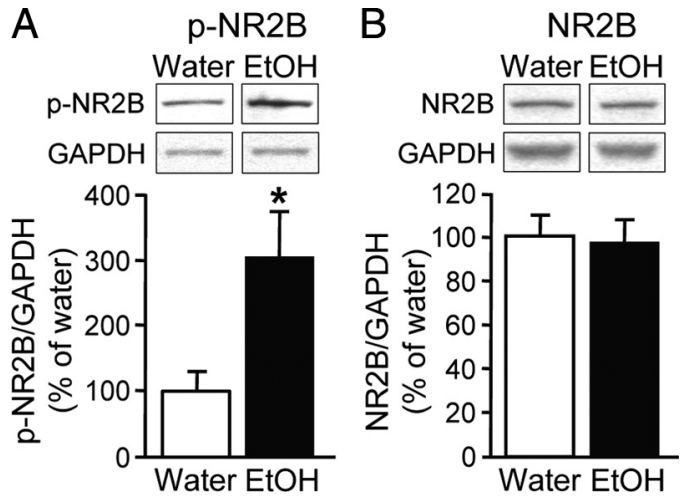

Figure 7. Intermittent access to ethanol results in increased phosphorylation of NR2B subunits in the DMS. Rats were exposed to 18 drinking sessions using the intermittent access to $20 \%$ ethanol two-bottle choice procedure. One day after the last ethanol-drinking session, DMS tissue was dissected out and protein and phosphorylation levels of NR2B subunits in homogenates were measured. $\boldsymbol{A}$, Intermittent access to ethanol results in increased phosphorylation levels of NR2B subunits in the DMS. Phospho-NR2B levels were measured using anti[pY1472]NR2B ( $p$-NR2B) antibodies (top). Bar graphs summarizing the averaged changes in phosphorylation levels of NR2B subunits (bottom). Western blot data were normalized to GAPDH and plotted as percentage of water control. ${ }^{*} p<0.05$ versus water (two-tailed $t$ test). $n=7$ (water). $n=8$ (EtOH). $\boldsymbol{B}$, Intermittent access to ethanol does not alter the protein levels of NR2B subunits in total DMS homogenates. Top, Image is representative of $n=7$ (water) and 8 (EtOH). Bottom, Bar graphs summarizing the averaged changes in protein levels of NR2B subunits in total homogenates. $p>0.05$ EtOH versus water (two-tailed $t$ test).
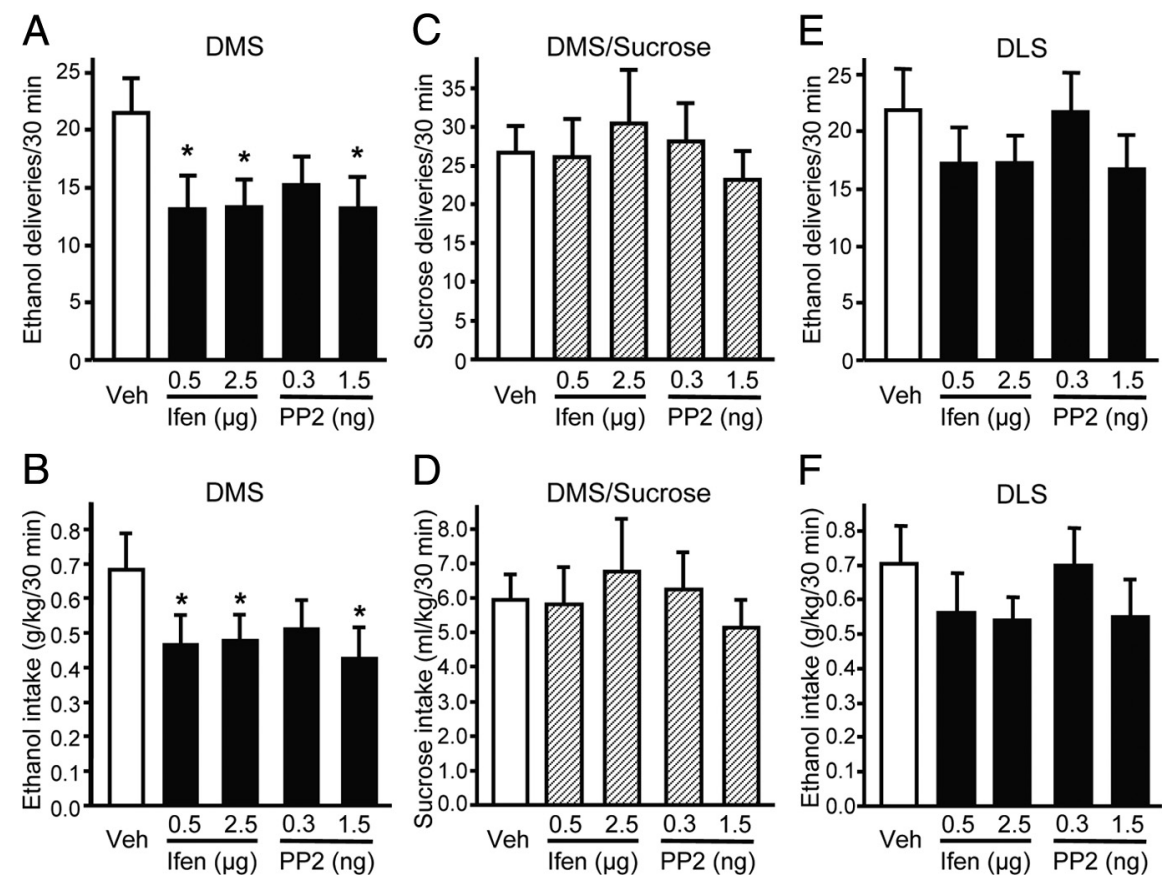

Figure 8. Inhibition of NR2B-NMDARs or Src PTKs in the DMS but not in the DLS decreases operant ethanol self-administration. Rats were infused with the NR2B-NMDAR antagonist ifenprodil (Ifen, 0.5 and $2.5 \mu \mathrm{g} / \mu \mathrm{l} / \mathrm{side}$ ), the Src PTK inhibitor PP2 ( 0.3 and $1.5 \mathrm{ng} / \mu \mathrm{l} /$ side) or vehicle (Veh) 15 min before the beginning of the test session in the DMS $(\boldsymbol{A}-\boldsymbol{D})$ or in the DLS $(\boldsymbol{E}, \boldsymbol{F}) . \boldsymbol{A}, \boldsymbol{E}$, Mean \pm SEM of the number of ethanol deliveries during a 30 min session. $n=6-7 .{ }^{*} p<0.05$ versus Veh. $\boldsymbol{B}, \boldsymbol{F}$, Mean \pm SEM of the number of ethanol intake (in grams per kilogram) in rats trained on an FR3 schedule to obtain $0.1 \mathrm{ml}$ of a $20 \%$ ethanol solution per delivery during 30 min sessions in $\boldsymbol{A}$ and $\boldsymbol{E}$, respectively. $n=6-7 .{ }^{*} p<0.05$ versus Veh. $\boldsymbol{C}$, Mean \pm SEM of the number of sucrose deliveries during a 30 min session. $n=8 . D$, Mean \pm SEM of the number of deliveries in rats trained on an FR3 schedule to obtain $0.1 \mathrm{ml}$ of a $2.5 \%$ sucrose solution per delivery during 30 min sessions in $C . n=8$.

icantly affect operant ethanol self-administration [Fig. $8 E$ (no significant effect of the treatment: $\left.F_{(4,24)}=1.27, p=0.31\right)$ and Fig. $8 F$ (no significant effect of the treatment: $F_{(4,24)}=1.01, p=$ $0.38)]$. These data suggest that long-term activation of Fyn and 

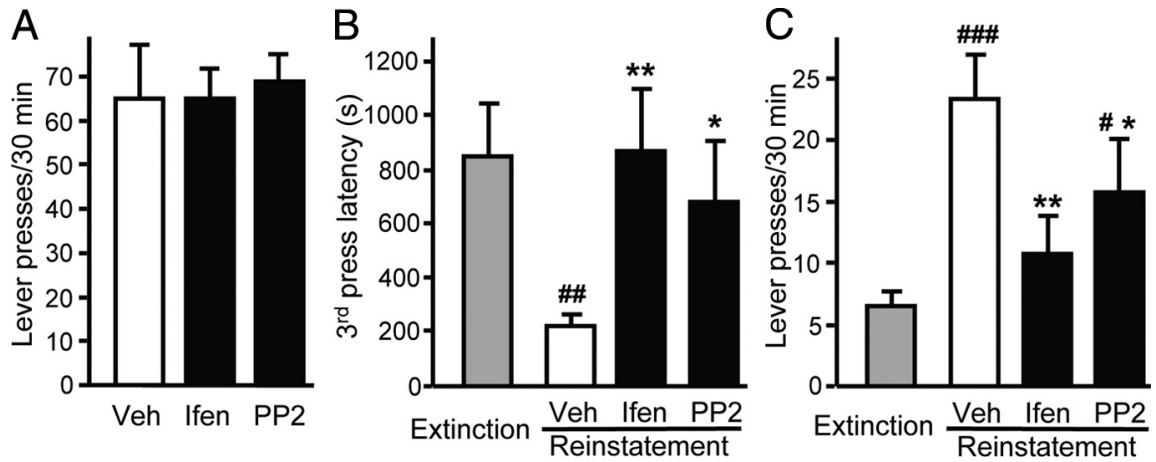

Figure 9. Inhibition of NR2B-NMDARs or Src PTKs in the DMS reduces ethanol-priming-induced reinstatement of ethanol seeking. Ifenprodil $(2.5 \mu \mathrm{g} / \mu \mathrm{l} / \mathrm{side}), \mathrm{PP} 2(1.5 \mathrm{ng} / \mu \mathrm{l} / \mathrm{side})$, or vehicle was infused $15 \mathrm{~min}$ before the beginning of the test session. $A$, Mean \pm SEM of the number of presses during a 30 min test in extinction. $n=7 . B$, Mean \pm SEM of latency in seconds to the third press (first reward) during the final extinction session and the reinstatement test. $C$, Mean \pm SEM of the number of presses during the final extinction session and reinstatement of ethanol seeking induced by an oral ethanol prime. $B, C, n=8 .{ }^{\#} p<0.05$, ${ }^{\#} p<0.01$, ${ }^{\# \#} p<0.001$ versus extinction ${ }^{*} p<0.05,{ }^{* *} p<0.01$, treatment versus Veh.

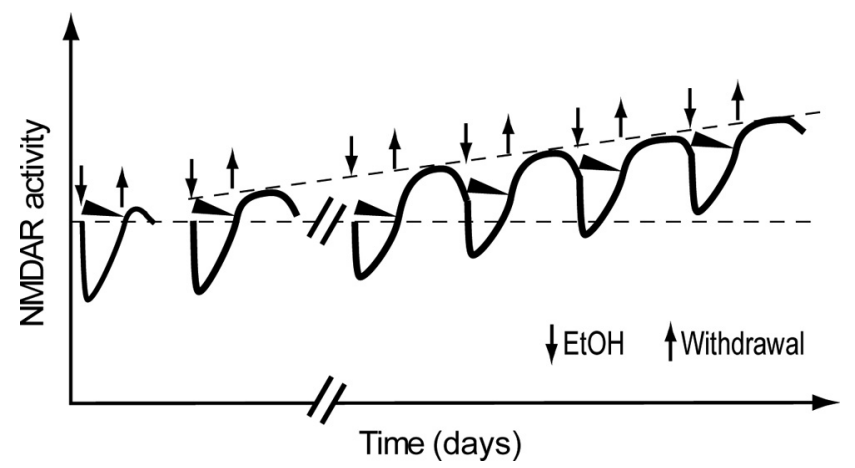

Figure 10. Hypothetical model of the mechanism leading to increased NR2B-NMDAR activity during the withdrawal period following repeated ethanol exposure. Initial exposure of DMS neurons to ethanol results in a rapid inhibition of the activity of the NR2BNMDAR channel; however, ethanol withdrawal leads to a long-lasting facilitation of channel activity. Repeated exposure of the DMS to ethanol results in a gradual reduction of the inhibitory action of ethanol on the channel and increased facilitation of the activity of the channel following withdrawal. These changes are likely to be due to long-lasting activation of Fyn kinase, long-lasting phosphorylation of NR2B, and forward trafficking of the subunit to the synaptic membrane.

NR2B-NMDARs within the DMS are important players in the mechanism that underlies excessive ethanol-drinking behavior.

\section{Inhibition of Src PTKs or NR2B-NMDARs in the DMS reduces reinstatement of ethanol seeking in rats}

To test whether the decrease in operant ethanol selfadministration induced by the inhibition of Src PTKs activity or NR2B-NMDARs is due to a decrease in the motivation of rats to seek ethanol, instrumental performances were evaluated during a single session of extinction. As shown in Figure 9A, intra-DMS infusion of ifenprodil and PP2 at a dose that efficiently reduced operant ethanol self-administration (Fig. 8A) did not decrease the number of lever presses on the ethanol lever during the extinction session (no significant effect of treatment: $F_{(2,12)}=$ $0.075, p=0.93$ ). We also tested whether intra-DMS infusion of ifenprodil or PP2 affects ethanol-priming-induced reinstatement of ethanol seeking as described in Materials and Methods. The initiation of operant responding during the reinstatement test, as assessed by the latency to the third press (i.e., the first reward), was reduced in vehicle-treated rats compared to the last day of extinction (Fig. 9B) (significant effect of treatment: $\chi^{2}=10.35$, $p<0.02$ ), indicating that the oral ethanol prime efficiently reinstated ethanol seeking. However, this priming effect was prevented by intra-DMS infusion of ifenprodil or PP2 (Fig. 9B). Consistent with the result above, reinstatement of ethanol seeking was significantly reduced by intra-DMS infusion of ifenprodil or PP2 (Fig. 9C) (significant effect of treatment: $\left.F_{(3,21)}=8.15, p<0.001\right)$. Therefore, these data indicate that the NR2B subunit and Fyn are major components of the mechanisms controlling reinstatement of ethanol seeking by an ethanol prime.

\section{Discussion}

Here we demonstrate that repeated systemic administration of ethanol as well as intermittent excessive ethanol consumption produces a robust long-lasting increase in the activity of the synaptic NMDAR in the DMS. We further provide evidence to suggest that Fyn-mediated phosphorylation of the NR2B subunit, which in turn results in higher levels of the NR2B-NMDARs at the synaptic membrane, could be the mechanism that underlies the long-lasting upregulation of NR2B-NMDAR activity in response to ethanol. Finally, we show that the inhibition of NR2B-NMDARs or the Src PTKs (which include Fyn) in the DMS decreases operant ethanol self-administration and reinstatement of ethanol seeking in rats that consume high levels of ethanol.

\section{Long-term facilitation of the NR2B-NMDAR function upon} ethanol exposure and withdrawal is centered in the DMS Ethanol-mediated LTF of NMDAR activity was not observed in the DLS, although the protein levels of NR2A and NR2B subunits and Fyn (data not shown) in the DMS and DLS and the synaptic responses in the two subregions of naive rats were similar. An alternative mechanism for the subregion specificity may be linked to whether or not Fyn is associated to the NMDAR-NR2B complex. Previously, we observed that ex vivo and in vivo exposure to ethanol leads to phosphorylation of NR2B subunit in the dorsal striatum where the NR2B subunit and Fyn are colocalized, but not in the ventral striatum where NR2B and Fyn are not compartmentalized within the same complex (Wang et al., 2007). Therefore, the compartmentalization of Fyn near the NMDAR complex in the DMS might account for the enhanced LTF in the DMS compared to the DLS.

The activity of DMS NMDARs following ex vivo acute withdrawal from ethanol was also examined by Yin et al. (2007), but no facilitation was observed following ethanol withdrawal. While the discrepancy between our studies and that of Yin et al. (2007) is unclear, the studies are difficult to compare since Yin et al. (2007) relied only on ex vivo slices electrophysiology experiments, and the experimental conditions were different. For example, Yin et al. (2007) used a higher concentration of EGTA in the intracellular solution, which may buffer the $\mathrm{Ca}^{2+}$ rise that is required for acute ethanol withdrawal-induced facilitation of NMDAR activity (Li et al., 2005). Another possibility is the inclusion of a tyrosine phosphatase inhibitor during recording in our study. 


\section{Long-lasting upregulation of NMDAR activity in the DMS upon voluntary and passive exposure to ethanol}

We found that repeated systemic administration or voluntary consumption of ethanol results in a long-lasting upregulation of the activity of synaptic NMDARs in the DMS. This result is particularly striking as the increase in synaptic NMDAR activity remains high even $9 \mathrm{~d}$ after the last episode of ethanol drinking. We further showed that repeated exposure to ethanol leads to an increase in the contribution of the NR2B subunit to the overall activity of the channel. Together, our results suggest that the NR2B-dependent LTF may underlie the persistent increase in the function of the NR2B-NMDAR induced by repeated in vivo ethanol exposure. Based on these results, we suggest the following model: the first administration of ethanol induces LTF of the NR2B-NMDAR activity that decays over time (Fig. 10). However, repeated cycles of ethanol exposure and withdrawal results in a cumulative enhancement of the NR2B-NMDAR activity (Fig. 10).

Interestingly, the upregulation in the activity of the channel in the DMS upon multiple ethanol exposures corresponded with a striking long-lasting increase in the activity of Fyn, as well as in the phosphorylation of its substrate the NR2B subunit, and a corresponding increase in the localization of the subunit in the synaptosomal compartment. Importantly, voluntary consumption of ethanol also produced an increase in the phosphorylation of NR2B, which was not due to an increase in the protein level of the subunit. Fyn phosphorylation of the NR2B subunit was shown to enhance the activity of the channel (Yaka et al., 2002, 2003), to promote the trafficking of the receptor from internal pools and to increase its stability at the cell surface (Nakazawa et al., 2001; Dunah et al., 2004; Prybylowski et al., 2005; Hayashi et al., 2009). Therefore, a long-lasting and cumulative elevation in Fyn activity in vivo may underlie the increased NR2B-NMDAR function in the DMS induced by repeated ethanol exposure.

\section{NR2B-NMDARs and Fyn in the DMS contribute to mechanisms that underlie excessive ethanol consumption and relapse}

The intermittent access two-bottle choice drinking procedure induces a progressive increase in ethanol intake and preference in rats (Carnicella et al., 2009) that resembles binge drinking in humans (National Institute on Alcohol Abuse and Alcoholism, 2004). We found that intra-DMS, but not intra-DLS, infusion of the NR2B-NMDAR antagonist ifenprodil significantly reduces operant ethanol self-administration in rats with a history of excessive voluntary ethanol consumption. Similar results were obtained upon the inhibition of Src PTKs activity in the DMS with PP2. Furthermore, infusion of ifenprodil or PP2 in the DMS does not alter operant sucrose self-administration, indicating that the inhibition of NR2B-NMDARs or Src PTKs interferes with processes specifically induced by ethanol that are not shared with non-drug reinforcing substances. We previously reported that LTF of NMDAR-mediated EPSCs in response to ex vivo exposure of DS slices to ethanol is not observed in Fyn deletion mice (Wang et al., 2007), and that Src activity is inhibited after acute treatment of ethanol in hippocampal slices (Suvarna et al., 2005). Therefore, although PP2 is not a selective Fyn kinase inhibitor, it is highly likely that the inhibitory actions of PP2 on ethanol-drinking behaviors are due to the inhibition of Fyn. Therefore, these results suggest that the long-lasting adaptive upregulation of Fyn and NR2B-NMDAR activity in the DMS observed upon repeated ethanol exposure contributes to mechanisms underlying excessive ethanol-drinking and -seeking behaviors.
We found that infusion of ifenprodil or PP2 into the DMS decreases reinstatement of ethanol seeking induced by re-exposure of rats to the exteroceptive properties of ethanol. This finding is reminiscent of the role of the DMS in the processing of A-O actions and the control of instrumental performance by the outcome, which mediate goal-directed behaviors (Yin and Knowlton, 2006; Balleine et al., 2009). In line with these findings, Vengeliene et al. (2005) showed that systemic administration of ifenprodil attenuates the alcohol deprivation effect, another model of relapse, in rats. Importantly, we also show that the NMDAR activity remains elevated in the DMS $9 \mathrm{~d}$ after the last ethanol-drinking session, which corresponds with the reinstatement test day. Together, these results suggest that the DMS is a critical substrate for relapse mechanisms involving the NR2B-NMDAR.

\section{NR2B-NMDARs in the DMS and mechanisms that underlie ethanol consumption, seeking, and relapse}

Increasing evidence suggests that aberrant neuroadaptations induced by drugs of abuse in the dorsal striatum result in compulsive drug seeking and taking (Gerdeman et al., 2003; Everitt and Robbins, 2005; Everitt et al., 2008; Belin et al., 2009). However, little is known about the molecular and cellular mechanisms implicated in such modifications. NMDARs are crucial for synaptic plasticity (Malenka and Nicoll, 1993), and our results suggest that aberrant synaptic plasticity in the DMS resulting from ethanol exposure contributes to the development and/or maintenance of ethanol-drinking and -seeking behaviors. Several possibilities may underlie the contribution of the NMDAR in the DMS to ethanol consumption and relapse. First, LTP of AMPARs in the DMS is NMDAR dependent (Partridge et al., 2000), and very recently Foster et al. (2010) showed that the NR2B subunit is essential for LTP in the hippocampus. Therefore, it is plausible that the facilitation of NR2B-NMDAR activity reduces the threshold of AMPAR-LTP induction. LTP in turn may strengthen goaldirected behaviors associated with ethanol. Another possibility relies on the observation that NMDAR activity contributes "UP state" firing of striatal neurons (Pomata et al., 2008). Specifically, the medium spiny neurons (MSNs), which are the principal cells of the striatum, alternate between two states: the depolarized UP state and more hyperpolarized DOWN state (Nicola et al., 2000). MSNs fire action potentials only during the UP state (Mahon et al., 2001; Tseng et al., 2001; Kasanetz et al., 2008). Therefore, it is possible that repeated cycles of ethanol exposure and withdrawal during the intermittent-access procedure induce increased NR2B-NMDAR activity, which in turn leads to increased UP state potentials of the neurons in the DMS. This change in neuronal activity results in a reduction in afferent threshold for neuronal firing action potentials, and consequently contributes to increased ethanol seeking and intake. Last, NMDAR activity may also contribute directly to the fast synaptic transmission, which is mediated by AMPARs under presynaptic stimulation (Miwa et al., 2008), and can directly contribute to the information flow through the striatum.

In summary, our results suggest that the Fyn/NR2B-NMDAR pathway in the DMS plays an important role in neuroadaptations that lead to excessive alcohol consumption and to the development of compulsive alcohol seeking. Our study also puts forward an important role of the NMDARs in the DMS in the propensity to relapse.

\section{References}

Balleine BW, Delgado MR, Hikosaka O (2007) The role of the dorsal striatum in reward and decision-making. J Neurosci 27:8161-8165. 
Balleine BW, Liljeholm M, Ostlund SB (2009) The integrative function of the basal ganglia in instrumental conditioning. Behav Brain Res 199:43-52.

Belin D, Jonkman S, Dickinson A, Robbins TW, Everitt BJ (2009) Parallel and interactive learning processes within the basal ganglia: relevance for the understanding of addiction. Behav Brain Res 199:89-102.

Carnicella S, Kharazia V, Jeanblanc J, Janak PH, Ron D (2008) GDNF is a fast-acting potent inhibitor of alcohol consumption and relapse. Proc Natl Acad Sci U S A 105:8114-8119.

Carnicella S, Amamoto R, Ron D (2009) Excessive alcohol consumption is blocked by glial cell line-derived neurotrophic factor. Alcohol 43:35-43.

Carpenter-Hyland EP, Woodward JJ, Chandler LJ (2004) Chronic ethanol induces synaptic but not extrasynaptic targeting of NMDA receptors. J Neurosci 24:7859-7868.

Carvelli L, Morón JA, Kahlig KM, Ferrer JV, Sen N, Lechleiter JD, LeebLundberg LM, Merrill G, Lafer EM, Ballou LM, Shippenberg TS, Javitch JA, Lin RZ, Galli A (2002) PI 3-kinase regulation of dopamine uptake. J Neurochem 81:859-869.

Chaudhri N, Sahuque LL, Cone JJ, Janak PH (2008) Reinstated ethanolseeking in rats is modulated by environmental context and requires the nucleus accumbens core. Eur J Neurosci 28:2288-2298.

Corbit LH, Janak PH (2007) Inactivation of the lateral but not medial dorsal striatum eliminates the excitatory impact of Pavlovian stimuli on instrumental responding. J Neurosci 27:13977-13981.

Daws LC, Callaghan PD, Morón JA, Kahlig KM, Shippenberg TS, Javitch JA, Galli A (2002) Cocaine increases dopamine uptake and cell surface expression of dopamine transporters. Biochem Biophys Res Commun 290:1545-1550.

Dickinson A, Wood N, Smith JW (2002) Alcohol seeking by rats: action or habit? Q J Exp Psychol B 55:331-348.

Dunah AW, Standaert DG (2001) Dopamine D1 receptor-dependent trafficking of striatal NMDA glutamate receptors to the postsynaptic membrane. J Neurosci 21:5546-5558.

Dunah AW, Sirianni AC, Fienberg AA, Bastia E, Schwarzschild MA, Standaert DG (2004) Dopamine D1-dependent trafficking of striatal N-methylD-aspartate glutamate receptors requires Fyn protein tyrosine kinase but not DARPP-32. Mol Pharmacol 65:121-129.

Everitt BJ, Robbins TW (2005) Neural systems of reinforcement for drug addiction: from actions to habits to compulsion. Nat Neurosci 8:1481-1489.

Everitt BJ, Belin D, Economidou D, Pelloux Y, Dalley JW, Robbins TW (2008) Review. Neural mechanisms underlying the vulnerability to develop compulsive drug-seeking habits and addiction. Philos Trans R Soc Lond B Biol Sci 363:3125-3135.

Foster KA, McLaughlin N, Edbauer D, Phillips M, Bolton A, ConstantinePaton M, Sheng M (2010) Distinct roles of NR2A and NR2B cytoplasmic tails in long-term potentiation. J Neurosci 30:2676-2685.

Gerdeman GL, Ronesi J, Lovinger DM (2002) Postsynaptic endocannabinoid release is critical to long-term depression in the striatum. Nat Neurosci 5:446-451.

Gerdeman GL, Partridge JG, Lupica CR, Lovinger DM (2003) It could be habit forming: drugs of abuse and striatal synaptic plasticity. Trends Neurosci 26:184-192.

Hallett PJ, Spoelgen R, Hyman BT, Standaert DG, Dunah AW (2006) Dopamine D1 activation potentiates striatal NMDA receptors by tyrosine phosphorylation-dependent subunit trafficking. J Neurosci 26:46904700.

Hayashi T, Thomas GM, Huganir RL (2009) Dual palmitoylation of NR2 subunits regulates NMDA receptor trafficking. Neuron 64:213-226.

Hohmann AG, Herkenham M (2000) Localization of cannabinoid CB(1) receptor mRNA in neuronal subpopulations of rat striatum: a doublelabel in situ hybridization study. Synapse 37:71-80.

Hyman SE, Malenka RC, Nestler EJ (2006) Neural mechanisms of addiction: the role of reward-related learning and memory. Annu Rev Neurosci 29:565-598.

Jeanblanc J, He DY, Carnicella S, Kharazia V, Janak PH, Ron D (2009) Endogenous BDNF in the dorsolateral striatum gates alcohol drinking. J Neurosci 29:13494-13502.

Joel D, Weiner I (2000) The connections of the dopaminergic system with the striatum in rats and primates: an analysis with respect to the functional and compartmental organization of the striatum. Neuroscience 96:451-474.
Kasanetz F, Riquelme LA, Della-Maggiore V, O'Donnell P, Murer MG (2008) Functional integration across a gradient of corticostriatal channels controls UP state transitions in the dorsal striatum. Proc Natl Acad Sci U S A 105:8124-8129.

Li HF, Mochly-Rosen D, Kendig JJ (2005) Protein kinase Cgamma mediates ethanol withdrawal hyper-responsiveness of NMDA receptor currents in spinal cord motor neurons. Br J Pharmacol 144:301-307.

Mahon S, Deniau JM, Charpier S (2001) Relationship between EEG potentials and intracellular activity of striatal and cortico-striatal neurons: an in vivo study under different anesthetics. Cereb Cortex 11:360-373.

Malenka RC, Nicoll RA (1993) NMDA-receptor-dependent synaptic plasticity: multiple forms and mechanisms. Trends Neurosci 16:521-527.

McGeorge AJ, Faull RL (1989) The organization of the projection from the cerebral cortex to the striatum in the rat. Neuroscience 29:503-537.

Miles FJ, Everitt BJ, Dickinson A (2003) Oral cocaine seeking by rats: action or habit? Behav Neurosci 117:927-938.

Miwa H, Fukaya M, Watabe AM, Watanabe M, Manabe T (2008) Functional contributions of synaptically localized NR2B subunits of the NMDA receptor to synaptic transmission and long-term potentiation in the adult mouse CNS. J Physiol 586:2539-2550.

Monyer H, Sprengel R, Schoepfer R, Herb A, Higuchi M, Lomeli H, Burnashev N, Sakmann B, Seeburg PH (1992) Heteromeric NMDA receptors: molecular and functional distinction of subtypes. Science 256:1217-1221.

Nakazawa T, Komai S, Tezuka T, Hisatsune C, Umemori H, Semba K, Mishina M, Manabe T, Yamamoto T (2001) Characterization of Fynmediated tyrosine phosphorylation sites on GluR epsilon 2 (NR2B) subunit of the N-methyl-D-aspartate receptor. J Biol Chem 276:693-699.

National Institute on Alcohol Abuse and Alcoholism (2004) NIAAA Council approves definition of binge drinking. NIAAA Newsletter, No 3 (NIH pub no 04-5346). Bethesda MD: National Institute on Alcohol Abuse and Alcoholism.

Nelson A, Killcross S (2006) Amphetamine exposure enhances habit formation. J Neurosci 26:3805-3812.

Nicola SM, Surmeier J, Malenka RC (2000) Dopaminergic modulation of neuronal excitability in the striatum and nucleus accumbens. Annu Rev Neurosci 23:185-215.

Nordquist RE, Voorn P, de Mooij-van Malsen JG, Joosten RN, Pennartz CM, Vanderschuren LJ (2007) Augmented reinforcer value and accelerated habit formation after repeated amphetamine treatment. Eur Neuropsychopharmacol 17:532-540.

O’Doherty J, Dayan P, Schultz J, Deichmann R, Friston K, Dolan RJ (2004) Dissociable roles of ventral and dorsal striatum in instrumental conditioning. Science 304:452-454.

Palop JJ, Chin J, Roberson ED, Wang J, Thwin MT, Bien-Ly N, Yoo J, Ho KO, Yu GQ, Kreitzer A, Finkbeiner S, Noebels JL, Mucke L (2007) Aberrant excitatory neuronal activity and compensatory remodeling of inhibitory hippocampal circuits in mouse models of Alzheimer's disease. Neuron 55:697-711.

Partridge JG, Tang KC, Lovinger DM (2000) Regional and postnatal heterogeneity of activity-dependent long-term changes in synaptic efficacy in the dorsal striatum. J Neurophysiol 84:1422-1429.

Paxinos G, Watson C (2007) The rat brain in stereotaxic coordinates, Ed 4. San Diego: Academic.

Pomata PE, Belluscio MA, Riquelme LA, Murer MG (2008) NMDA receptor gating of information flow through the striatum in vivo. J Neurosci 28:13384-13389.

Prybylowski K, Chang K, Sans N, Kan L, Vicini S, Wenthold RJ (2005) The synaptic localization of NR2B-containing NMDA receptors is controlled by interactions with PDZ proteins and AP-2. Neuron 47:845-857.

Qiang M, Denny AD, Ticku MK (2007) Chronic intermittent ethanol treatment selectively alters $\mathrm{N}$-methyl-D-aspartate receptor subunit surface expression in cultured cortical neurons. Mol Pharmacol 72:95-102.

Reep RL, Cheatwood JL, Corwin JV (2003) The associative striatum: organization of cortical projections to the dorsocentral striatum in rats. J Comp Neurol 467:271-292.

Standaert DG, Testa CM, Young AB, Penney JB Jr (1994) Organization of $\mathrm{N}$-methyl-D-aspartate glutamate receptor gene expression in the basal ganglia of the rat. J Comp Neurol 343:1-16.

Suvarna N, Borgland SL, Wang J, Phamluong K, Auberson YP, Bonci A, Ron D (2005) Ethanol alters trafficking and functional N-methyl-Daspartate receptor NR2 subunit ratio via H-Ras. J Biol Chem 280:31450-31459. 
Tanaka SC, Balleine BW, O’Doherty JP (2008) Calculating consequences: brain systems that encode the causal effects of actions. J Neurosci 28:6750-6755

Tricomi E, Balleine BW, O’Doherty JP (2009) A specific role for posterior dorsolateral striatum in human habit learning. Eur $J$ Neurosci 29:2225-2232.

Tricomi EM, Delgado MR, Fiez JA (2004) Modulation of caudate activity by action contingency. Neuron 41:281-292.

Tseng KY, Kasanetz F, Kargieman L, Riquelme LA, Murer MG (2001) Cortical slow oscillatory activity is reflected in the membrane potential and spike trains of striatal neurons in rats with chronic nigrostriatal lesions. J Neurosci 21:6430-6439.

Vengeliene V, Bachteler D, Danysz W, Spanagel R (2005) The role of the NMDA receptor in alcohol relapse: a pharmacological mapping study using the alcohol deprivation effect. Neuropharmacology 48:822-829.

Voorn P, Vanderschuren LJ, Groenewegen HJ, Robbins TW, Pennartz CM (2004) Putting a spin on the dorsal-ventral divide of the striatum. Trends Neurosci 27:468-474.

Wang J, Yeckel MF, Johnston D, Zucker RS (2004) Photolysis of postsynaptic caged $\mathrm{Ca} 2+$ can potentiate and depress mossy fiber synaptic responses in rat hippocampal CA3 pyramidal neurons. J Neurophysiol 91:15961607.

Wang J, Carnicella S, Phamluong K, Jeanblanc J, Ronesi JA, Chaudhri N, Janak PH, Lovinger DM, Ron D (2007) Ethanol induces long-term fa- cilitation of NR2B-NMDA receptor activity in the dorsal striatum: implications for alcohol drinking behavior. J Neurosci 27:3593-3602.

Weiss F, Lorang MT, Bloom FE, Koob GF (1993) Oral alcohol selfadministration stimulates dopamine release in the rat nucleus accumbens: genetic and motivational determinants. J Pharmacol Exp Ther 267:250-258.

Wise RA (1973) Voluntary ethanol intake in rats following exposure to ethanol on various schedules. Psychopharmacologia 29:203-210.

Yaka R, Thornton C, Vagts AJ, Phamluong K, Bonci A, Ron D (2002) NMDA receptor function is regulated by the inhibitory scaffolding protein, RACK1. Proc Natl Acad Sci U S A 99:5710-5715.

Yaka R, Phamluong K, Ron D (2003) Scaffolding of Fyn kinase to the NMDA receptor determines brain region sensitivity to ethanol. J Neurosci 23:3623-3632.

Yin HH, Knowlton BJ (2006) The role of the basal ganglia in habit formation. Nat Rev Neurosci 7:464-476.

Yin HH, Park BS, Adermark L, Lovinger DM (2007) Ethanol reverses the direction of long-term synaptic plasticity in the dorsomedial striatum. Eur J Neurosci 25:3226-3232.

Zapata A, Gonzales RA, Shippenberg TS (2006) Repeated ethanol intoxication induces behavioral sensitization in the absence of a sensitized accumbens dopamine response in C57BL/6J and DBA/2J mice. Neuropsychopharmacology 31:396-405. 